\title{
Biostatistical evidence for two distinct chronic open angle glaucoma populations
}

\author{
Michael Schulzer, Stephen M Drance, Cedric J Carter, Donald E Brooks, Gordon R Douglas, \\ Wing Lau
}

\begin{abstract}
Twenty-six eyes of 26 patients with low-tension glaucoma and 34 eyes of 34 patients with hightension glaucoma were studied. Fifty-one measurements were available on each patient, including visual field indices, finger blood flow measurements, as well as haematological, coagulation, and biochemical and rheological variables. Multivariate analysis revealed two statistically distinct groups of patients, with low and high tension glaucoma cases equally distributed in both. The smaller group (15 patients) showed a suggestion of vasospastic finger blood flow measurements, and had a high positive correlation between the mean deviation (MD) index of field severity and the highest intraocular pressure $(r=0.715$, $p=0.0008)$. The second, larger group (45 patients) showed disturbed coagulation and biochemical measurements, suggestive of vascular disease, and had no correlation between the MD index and the highest intraocular pressure.
\end{abstract}

There is increasing evidence that there may be multiple mechanisms of damage in the glaucomas. Clinically, some patients seem to derive benefit from reduction of intraocular pressure, while others continue to fail in spite of it. ${ }^{1-4} \mathrm{~A}$ number of arguments in support of more than one pathogenetic process have been outlined. ${ }^{56}$ Investigation into the nature of these processes is hampered by the difficulty in identifying clinically patients with specific mechanisms.

Potential heterogeneity in a population may

University of British

Columbia, Department of Statistics

M Schulzer

W Lau

Department of

Ophthalmology

$S M$ Drance

G R Douglas

Department of Medicine M Schulzer

Department of Pathology C J Carter

DE Brooks

Correspondence to:

Dr M Schulzer, Department of Medicine, Faculty of

Medicine, University of

British Columbia, 910 West

10th Avenue, Vancouver, BC,

Canada V5Z 4E3.

Accepted for publication

26 October 1989 procedure known as principal component analysis. The details of its methodology and applications are described elsewhere. ${ }^{7-9}$ This multivariate technique is used primarily to reduce large sets of correlated data to a relatively small number of uncorrelated 'components', which can in turn be analysed more readily with little loss of information. These components, calculated from the data, are weighted sums ('linear combinations') of the original measurements. The values of the various components for each subject are calculated and are referred to as 'scores'.

The principal components are computed sequentially from the data in the order of decreasing variability of their score values across subjects. Thus, in particular, the weights that the resulting scores will have the largest possible variation between individuals.

This feature of the principal component technique makes it a valuable tool for the detection of hidden clusters within a population, since such clusters, if they exist, are often best seen through the corresponding clustering of the score values of one or several of the principal components. Most commonly the first component, having the largest spread in its score values, is the most sensitive for the identification of potential clusters. Plots of scores in the first component for each subject against the corresponding scores in other components help to display the resulting clusters graphically. Statistical methods are used to determine the significance levels of apparent clusters.

Clearly, successful application of this method to the detection of a non-homogeneous population requires a representative sample and sufficient data on each subject to maximise the likelihood that relevant variables have been included.

\section{Materials and methods}

In 1986 we planned a study to examine the vascular, haematological, and rheological differences between patients diagnosed as having low-tension glaucoma (LTG) and those suffering from high-tension glaucoma (HTG). It was decided to examine approximately 50 consecutive cases of LTG and 50 cases of HTG. These patients were studied sequentially. Some of the patients were on topical therapy and others on no treatment. The patients came from our consultation service, and included also those patients whose disease was being monitored by us on an ongoing basis.

The LTG eyes had open angles, glaucomatous selected for the first component are calculated so abnormality of the optic discs, and characteristic nerve fibre bundle defects on Goldmann statickinetic perimetry or static perimetry on the Oculus perimeter or on automatic perimetry (30-2 Octopus or Humphrey). Their intraocular pressure was $\leqslant 21 \mathrm{mmHg}$ without therapy, including pressure studies round the clock. The HTG eyes had similar disc and field changes but pressures $\geqslant 22 \mathrm{mmHg}$.

In order to carry out a full-scale multivariate analysis only those patients on whom complete data (51 measurements) were available were included in this study. This led to a final sample of 26 LTG patients (mean age 61.9 (SD 12.2) years, mean intraocular pressure 19.3 (SD 1.8) $\mathrm{mmHg}$ ) and $34 \mathrm{HTG}$ patients (mean age $65 \cdot 5$ $(\mathrm{SD} 11 \cdot 5)$ years, mean intraocular pressure $28 \cdot 1$ 
$(\mathrm{SD} 4 \cdot 7) \mathrm{mmHg}$. One eye was randomly selected in each patient for the analysis.

The 51 measurements which were available on each patient included three visual field indices previously defined ${ }^{10}$ : the mean deviation (MD), which identifies the overall loss of sensitivity of points tested compared with their mean agerelated normal values; the corrected loss variation $(\mathrm{CLV})$, which describes the variation of threshold values between individual points tested, and provides early indication of localised field defects; and the short term fluctuation (SF), which measures the variation of all thresholds tested twice within a single field examination. In addition, the highest intraocular pressure (IOP), pupil size, visual acuity, and cup-disc ratio were recorded.

Systolic and diastolic blood pressures and electrocardiogram (ECG) (classified as normal or indicative of ischaemic abnormality) were obtained.

The blood flow in the finger and its response to heat and cold were measured by methods described elsewhere. ${ }^{11}$ Measurements were taken of the baseline flow, flow after heating (heated flow), flow after a 10-second exposure to cold (cold flow), as well as the ratios: heated flow/ baseline flow and heated flow/cold flow. The following haematological data were available: leucocyte count, red cell count, haemoglobin, haematocrit, mean corpuscular volume (MCV), mean corpuscular haemoglobin $(\mathrm{MCH})$, mean corpuscular haemoglobin concentration (MCHC), and platelet count.

In addition coagulation tests were performed after initial observations that low-tension glaucoma appeared to be associated with both cardiovascular and cerebrovascular disease, and that defective fibrinolysis was observed in patients with low-tension glaucoma. ${ }^{12}$ The tests chosen had been shown in previous prospective clinical studies to be associated with an increased risk of vascular disease. ${ }^{13}$ Tests included euglobulin lysis time (baseline and following ischaemic stress, and lysis time ratio of poststress to prestress), ${ }^{14}$ factor VII, factor VIII, fibrinogen, and antithrombin III.

Measurements were also available on haemoglobin A1C, 2-hour post/food (pc) glucose, blood urea nitrogen (BUN), creatinine, uric acid, total protein, albumin, $\alpha_{1}, \alpha_{2}, \beta, \gamma$ and total globulin, thyroxine $\left(\mathrm{T}_{4}\right)$, cholesterol, high density lipoprotein cholesterol (HDL), low density lipoprotein cholesterol (LDL), triglycerides, and the $\mathrm{HDL} /$ cholesterol ratio. Finally, rheological measurements included plasma viscosity and blood viscosity (peak and steady state).

\section{STATISTICAL METHODS}

The distribution of each of the 51 variables was examined. Distributions which failed to satisfy essential statistical assumptions (stability of variance, symmetry, normality) were identified, and a correcting logarithmic transformation was applied to the corresponding measurements for the purposes of the analysis. This included the following variables: corrected loss variation $(\mathrm{CLV})$, short term fluctuation (SF), visual acuity, cup-disc ratio, heated flow/baseline flow, heated flow/cold flow, euglobulin lysis time ratio, and $\mathrm{HDL} /$ cholesterol ratio.

A significant correlation was found between the mean deviation (MD) (see description above) and pupil size $(r=-0 \cdot 36, p=0 \cdot 0001)$. The mean deviation was therefore adjusted for pupil size by regression analysis.

Since many of the 51 measurements were clearly correlated, a correlation matrix was calculated separately for the LTG, the HTG, and the combined LTG-HTG groups.

For the purposes of analysis, the SAS principal component analysis package was used. The data were first 'standardised', by subtracting from each measurement its corresponding sample mean and dividing by its sample standard deviation. This device allowed all measurements to participate in the analysis in an equally useful way, by removing spurious effects due to different units of measurement and to unequal variances.

In the initial stage of the computation 51 principal components were calculated, each with 51 weights ('coefficients') for the 51 meaurements anaysed. To determine which of the weights were statistically significant, the corresponding standard error for each weight had to be calculated. These standard errors are not included in the output of most computer packages available at present, and were therefore programmed in by us using the mathematical methods outlined elsewhere.' In this way, by progressively removing less significant weights by a method referred to as 'backward elimination', the complexity of the data was gradually reduced, retaining only those measurements which made a significant contribution to the components. The aim was to remove redundant and overcorrelated variables without degrading the precision of the statistical fit, ultimately identifying potential clusters in the sample using a minimum number of informative and significant measurements (the 'principle of parsimony').

The analysis was carried out separately on the LTG and on the HTG subgroups, and then repeated across the combined LTG-HTG sample.

In addition, 'agglomerative' analysis was also performed on these patient groups. In this approach subsets of measurements (ophthalmic, haematological, biochemical, etc.) were separately analysed into principal components. The statistically significant variables collected from the measurement subsets were then combined ('agglomerated') in various ways into a single set, which was then analysed into its principal components and progressively pared down by 'backward elimination' as described above, to a minimal number of informative measurements.

Results were compared between the various approaches. In addition, cross validations were carried out by using a randomly selected half of the individuals in the sample as the "construction set', for which principal components were calculated, and then cross-testing the results on the remaining half of individuals in the 'validation set'.

Observed clusters were tested for the 
TABLE I Significant coefficients (weights) ${ }^{\star}$ in first principal component

\begin{tabular}{lcl}
\hline Variable & $\begin{array}{l}\text { Coefficient of } \\
\text { standardised variable }\end{array}$ & $\begin{array}{l}\text { Standard error } \\
\text { of coefficient }\end{array}$ \\
\hline Baseline flow & 0.323 & 0.065 \\
Cold flow & 0.333 & 0.063 \\
Log (hot flow/baseline flow) & -0.373 & 0.053 \\
Log (hot flow/cold flow) & -0.370 & 0.053 \\
Prestress euglobulin lysis time & 0.324 & 0.058 \\
Poststress euglobulin lysis time & 0.312 & 0.063 \\
Glucose & $0 \cdot 183$ & 0.072 \\
HDL & -0.286 & 0.069 \\
Triglycerides & 0.284 & 0.069 \\
Log (HDL/cholesterol) & -0.330 & 0.064 \\
\hline
\end{tabular}

* The 2-sided significance level of the glucose coefficient is 0.01 . All other coefficients are significant at $\mathrm{p}<0.001$.

† Variables were standardised by subtracting their sample means and dividing by their sample

standard deviations.

$\ddagger$ Logarithms were taken as natural logarithms

significance of the separation between them by $\chi^{2}$ test on their squared standard distance. ${ }^{9}$

Continuous and categorical variables were compared between the generated clusters by $t$ tests, $\chi^{2}$ tests, and methods of simple and multiple regression when appropriate.

\section{Results}

The findings reported are based on analyses on the pooled LTG-HTG sample. Results of analyses within the LTG and HTG groups were for the most part very similar and were well summarised by the pooled sample. The intraocular pressure measurement was excluded from the analyses given here, in order to avoid confounding with the LTG-HTG classification.

Following the scheme of analyses outlined in the 'Statistical methods' section, a set of 10 significant measurements was ultimately retained. Table I lists the variables and gives the weights of the first principal component for each
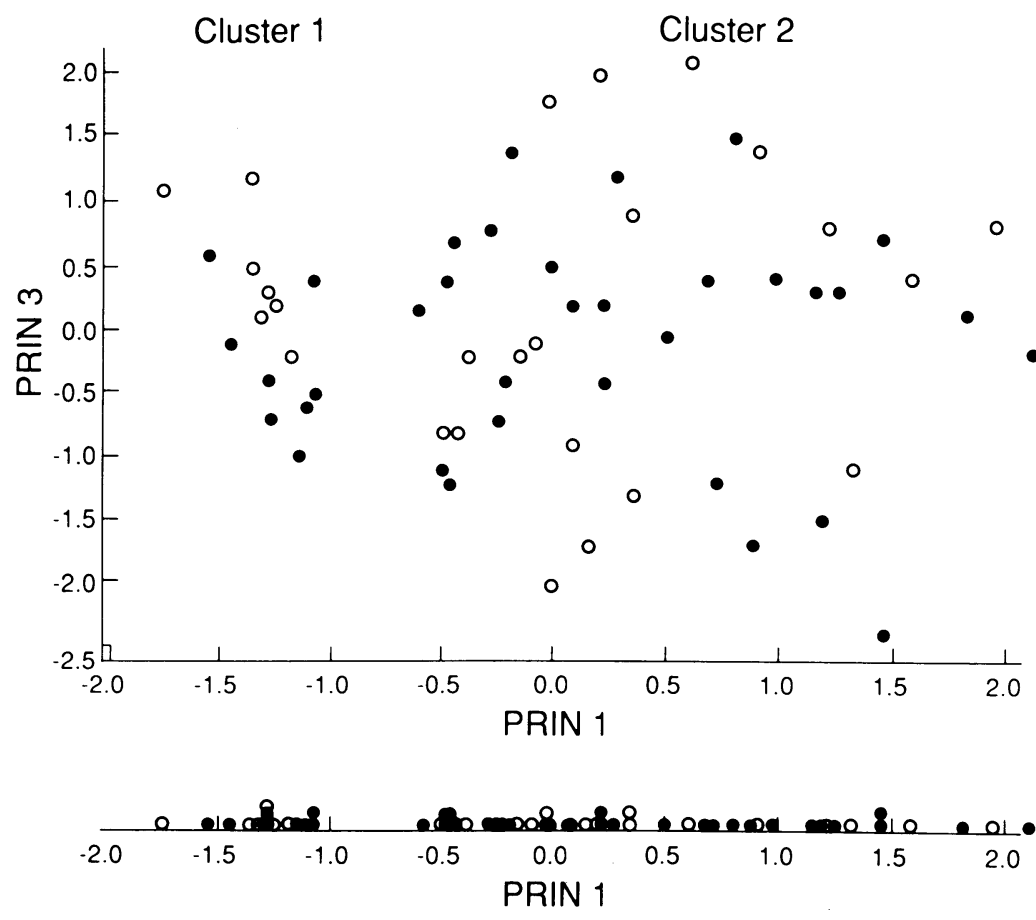

Figure 1: Scores of the first principal component (horizontal axis), summarising the data (51 observations) on 60 eyes of 60 patients, displayed for convenience both in a pointplot and in a scatterplot against corresponding scores of the third principal component (vertical axis). Black circles represent high-tension glaucoma eyes; open circles represent low-tension glaucoma eyes. A separation into two clusters (15 and 45 eyes respectively) can be observed. standardised variable, along with the corresponding estimated standard errors.

Fig 1 shows graphically the patient scores on the first principal component (horizontal axis), displayed as a pointplot, and as a scatterplot against the corresponding scores on the third principal component (vertical axis). Similar scatterplots were obtained relating all pairs of principal components. It is clear that the information regarding potential clusters is contained mainly in the first component. Two clusters are readily seen along the horizontal axis, separated at a score value of approximately $-0 \cdot 8$. The left-hand cluster (cluster 1 ) is small, consisting of 15 individuals, of whom 7 are LTG and 8 HTG. The large right-hand cluster (cluster 2) consists of 45 individuals, 19 LTG and 26 HTG. The proportions in each cluster of the LTG-HTG subgroups are clearly not significantly different $\left(\chi^{2}=0.09, \quad p=0.77\right)$. The observed clusters are statistically distinct $(p<0.0001)$. No other statistically significant clusters occur.

Table II gives the means and standard deviations in each cluster for the 10 variables identified in the final principal component, in unstandardised units. It also gives the means and standard deviations in each cluster for all other variables which showed a difference, significant at $\mathrm{p}<0 \cdot 10$, between the two cluster means. The statistical level of significance for each comparison is given. No significant differences were observed in mean age (64.1 (SD 15.7) years in cluster $1 ; 63.8(\mathrm{SD} 10 \cdot 4)$ years in cluster 2), mean highest intraocular pressure $(24 \cdot 7$ (SD $7 \cdot 0)$ versus $24 \cdot 2(\mathrm{SD} 5 \cdot 4) \mathrm{mmHg}$ ), mean pupil size $(3 \cdot 7(\mathrm{SD} 2 \cdot 3)$ versus $3 \cdot 3(\mathrm{SD} 1 \cdot 8) \mathrm{mm})$, mean systolic pressure $(117 \cdot 1$ (SD 18.8) versus $121 \cdot 2$ (SD $16 \cdot 2) \mathrm{mmHg}$ ) or in mean diastolic pressure $(74 \cdot 4(\mathrm{SD} 8 \cdot 1)$ versus $77 \cdot 9(\mathrm{SD} 10 \cdot 9) \mathrm{mmHg})$.

Cross-checking by other methods, as discussed earlier in the 'Methods' section, confirmed the separation into two disjoint clusters, yielding consistent results in terms of cluster variables and of cluster membership.

In order to examine the relationship between intraocular pressure and severity of the glaucomatous damage, simple linear regression of the pupil-adjusted mean deviation index (MD) of visual field severity on the highest - intraocular pressure (IOP) was carried out separately within each cluster. Fig 2 and 3 show the results. A very significant correlation was found in cluster $1 \quad(\mathrm{r}=0.715, \mathrm{p}=0.0008)$, indicating that the visual field loss, as measured by the $M D$, increased by $0.59 \mathrm{~dB}$ for each 1 $\mathrm{mmHg}$ increment in the highest IOP. No correlation was found in cluster $2(\mathrm{r}=0.043, \mathrm{p}=$ $0 \cdot 79)$.

The clusters were further analysed by multiple regressions to examine the joint relationship between mean deviation (MD), intraocular pressure (IOP), and corrected loss variation (CLV) (in log CLV units) in each.

In cluster 1 , where the MD and IOP were highly correlated, the MD and CLV were also significantly positively correlated $(p=0 \cdot 019)$ after both were adjusted for the IOP. On the other hand no relationship was found between the CLV and IOP after both had been adjusted 
TABLE II Means and standard deviations of variables in the two clusters $\star$

\begin{tabular}{|c|c|c|c|c|c|}
\hline \multirow[b]{2}{*}{ Variables } & \multicolumn{2}{|c|}{ Cluster 1} & \multicolumn{2}{|c|}{ Cluster 2} & \multirow[b]{2}{*}{$p^{t}$} \\
\hline & mean & $s d$ & mean & $s d$ & \\
\hline \multicolumn{6}{|l|}{ Cluster defining } \\
\hline Baseline flow & $5 \cdot 68$ & $4 \cdot 44$ & $31 \cdot 04$ & $20 \cdot 22$ & $<0.0001$ \\
\hline Cold flow & $4 \cdot 65$ & $3 \cdot 24$ & $16 \cdot 60$ & $13 \cdot 58$ & 0.0014 \\
\hline $\log (\text { hot flow/baseline flow })^{\ddagger}$ & $2 \cdot 62$ & 0.61 & 0.95 & 0.83 & $<0.0001$ \\
\hline Log (hot flow/cold flow) & $2 \cdot 80$ & 0.51 & $1 \cdot 57$ & 0.79 & $<0.0001$ \\
\hline Prestress euglobulin lysis time (minutes) & $253 \cdot 53$ & $83 \cdot 19$ & $513 \cdot 62$ & $216 \cdot 08$ & $<0.0001$ \\
\hline Poststress euglobulin lysis time (minutes) & $138 \cdot 00$ & $42 \cdot 63$ & 382.06 & $247 \cdot 75$ & 0.0004 \\
\hline $2 \mathrm{~h} \mathrm{pc}$ glucose $(\mathrm{mmol} / \mathrm{l})$ & $4 \cdot 42$ & $1 \cdot 44$ & $5 \cdot 80$ & $3 \cdot 24$ & 0.0280 \\
\hline $\mathrm{HDL}(\mathrm{mmol} / \mathrm{l})$ & $1 \cdot 40$ & $0 \cdot 34$ & 1.03 & $0 \cdot 27$ & $<0.0001$ \\
\hline Triglycerides $(\mathrm{mmol} / \mathrm{l})$ & 1.01 & $0 \cdot 35$ & $1 \cdot 82$ & $0 \cdot 88$ & 0.0010 \\
\hline Log (HDL/cholesterol) & 0.27 & $0 \cdot 07$ & $0 \cdot 18$ & 0.05 & $<0.0001$ \\
\hline \multicolumn{6}{|l|}{ Non-cluster-defining } \\
\hline $\mathrm{MD}(\mathrm{dB})$ & $12 \cdot 00$ & $5 \cdot 76$ & $8 \cdot 82$ & $5 \cdot 80$ & 0.0739 \\
\hline Fibrinogen $(\mathrm{g} / \mathrm{l})$ & $2 \cdot 81$ & 0.52 & $3 \cdot 13$ & 0.55 & 0.0520 \\
\hline WBC $\left(\times 10^{3}\right)$ & $4 \cdot 52$ & $1 \cdot 30$ & $5 \cdot 40$ & $1 \cdot 10$ & 0.0126 \\
\hline Uric acid $(\mu \mathrm{mol} / \mathrm{l})$ & 278.96 & $76 \cdot 13$ & $325 \cdot 95$ & $74 \cdot 35$ & 0.0419 \\
\hline Cholesterol $(\mathrm{mmol} / \mathrm{l})$ & $5 \cdot 24$ & 0.95 & $5 \cdot 78$ & 0.93 & 0.0592 \\
\hline $\mathrm{LDL}(\mathrm{mmol} / \mathrm{l})$ & $3 \cdot 29$ & $0 \cdot 73$ & $3 \cdot 85$ & 0.88 & 0.0309 \\
\hline ECG (proportion abnormal) & 0 & & 0.44 & & 0.0440 \\
\hline
\end{tabular}

^Included are all variables which showed a difference in the two cluster means at a 2-sided significance level of $\mathrm{p}<0 \cdot 10$.

$\dagger 2$-Sided $t$ test; for ECG, $\chi^{2}$ test.

$\mp$ Logs represent natural logarithms.

for the MD. The IOP and CLV together explained $70 \%$ of the variation in the MD measurements.

In cluster 2, where the MD and IOP were not correlated, a high correlation was observed between the $M D$ and $\operatorname{CLV}(r=0.70, p=0.0001)$. As in cluster 1 , no correlation could be found between the CLV and IOP.

The criterion for classifying a case into cluster 1 or 2 of Fig 1 is given in terms of raw (nonstandardised) measurement units (described in Table II) as follows: for a given case the following calculation should be made: ('log' means natural logarithm): $0.01556 \times$ baseline flow $+0.02578 \times$ cold flow $-0.34979 \times \log$ (hot flow/baseline flow) $-0.40977 \times \log$ (hot flow/cold flow) $+0.00146 \times$ prestress euglobulin lysis time $+0.00130 \times$ poststress euglobulin lysis time $+0.06233 \times 2 \mathrm{~h}$ pc glucose $-0.87780 \times$ high density lipoprotein cholesterol $+0.33127 \times$ triglycerides $-0.97802 \times$ $\log$ (HDL/cholesterol) $-0 \cdot 2072$.

When the resulting value of the criterion is negative, the case is classified into cluster 1 , while a positive value would lead to classification in cluster 2 . The criterion has been selected so as to minimise the chances of misclassification. In

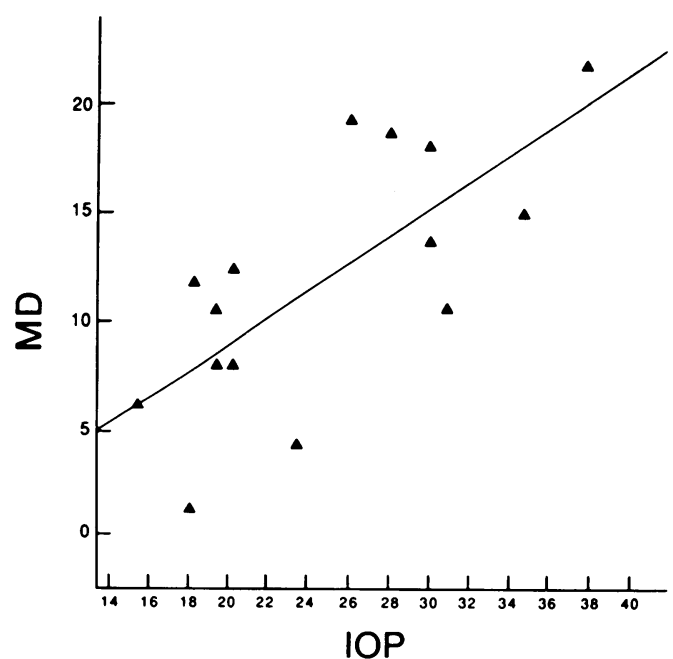

Figure 2: Scatterplot of mean deviation (MD) against highest intraocular pressure (IOP) in the 15 eyes of cluster 1 . The estimated regression line is highly significant $(r=0.715$, $p=0.0008$ ). fact, it is estimated that only approximately $4.3 \%$ of cluster 1 individuals might be misclassified into cluster 2 , and conversely, by this criterion.

\section{Discussion}

Principal component analysis is an efficient, guided method for detecting potential heterogeneity in a population. As applied to a pooled sample of 60 low-tension and high-tension glaucoma patients it produced two distinct clusters, consisting of 15 and 45 members respectively. This separation was accomplished independently of the highest intraocular pressure level, with both clusters containing nearly equal proportions of low- and hightension glaucoma patients. The two clusters were generated by the scores of the first principal component derived from 10 significant variables which were selected in stages from an initial set of 51. The final variables represented blood flow measurements in the finger under baseline conditions as well as after heating and cooling the hand, coagulation variables, and biochemical information.

The two clusters showed striking statistical

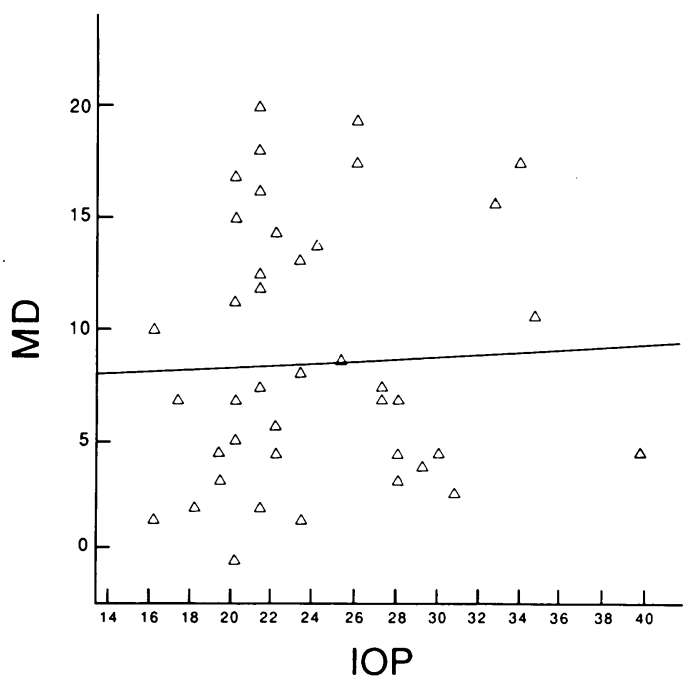

Figure 3: Scatterplot of mean deviation $(M D)$ against highest intraocular pressure (IOP) in the 45 eyes of cluster 2 . The estimated regression line is not significant $(r=0.043, p=0.79)$. 
differences. The smaller cluster (cluster 1) showed a lowered mean baseline blood flow level in the finger, and a lowered mean finger blood flow level after cooling, with corresponding increases in the means of the ratios of heated flow to baseline flow, and of heated flow to blood flow after cooling. These changes in blood flow in the finger in cluster 1 suggested vasospastic features. Cluster 1 showed no disturbances in any of the other measurements, and no ischaemic ECG abnormalities were noted in any individual in this cluster. The cluster was characterised by a remarkably high correlation between the degree of visual field disturbance, as measured by the mean deviation (MD), and the highest intraocular pressure of the eye (IOP) $(r=0.715$, $p=0.0008$ ). The larger cluster (cluster 2 ) showed no correlation whatever between the degree of visual field disturbance as reflected by the MD index and the highest intraocular pressure of the affected eyes. This cluster was characterised by raised mean euglobulin lysis times before and after ischaemic stress, with an increased poststress to prestress ratio, increased mean fibrinogen level, higher mean 2-h pc glucose level, a lower mean HDL and higher mean LDL levels, with a lower mean ratio of $\mathrm{HDL}$ to cholesterol, a higher mean level of triglycerides, a considerable rate of ischaemic ECG abnormalities, as well as a higher mean leucocyte count and a higher mean uric acid level. However, the mean finger blood flow measurements in this cluster were normal, and not of the vasospastic variety.

It appears that two distinct and statistically significantly different glaucoma subgroups have been identified, raising the possibility of different pathogenetic mechanisms: a smaller group, showing vasospastic trends in finger blood flow and a strong relationship between field disturbance and highest intraocular pressure; and a larger group, showing higher means in clotting and biochemical parameters and ECG anomalies suggestive of vascular disease. The latter group showed no relationship between degree of field disturbance and the highest intraocular pressure. Group separation has been accomplished without regard to the traditional arbitrary low-tension and hightension classification and seems to be altogether independent of the highest pressure level.

In spite of the consistency of these findings, and the support of cross validation analysis, caution in their implications must be recommended. Further testing of widely based data sets over large, representative samples cross sectionally and longitudinally needs to be undertaken to confirm and evaluate the clinical significance of these results.

This study was supported by Medical Research Council of Canada grant MT 1578

1 Smith R. Medical v surgical therapy in glaucoma patients. Doc Ophthalmol Proc Symp Ser. Medical therapy in glaucoma, Amsterdam 1976. The Hague: Junk, 1977: 12: 123-30.

2 Drance SM, Werner EB, Schulzer M. The effect of trabeculectomy on the progression of glaucomatous visual trabeculectomy on the progression of glauco
field loss. Arch Ophthalmol 1977; 95: 1374-7.

3 Hitchings RA. Low tension glaucoma - is treatment worthwhile? Eye 1988; 2: 636-40.

4 Sommer A. Intraocular pressure and glaucoma (editorial). $A m$ f Ophthalmol 1989; 107: 186-8.

5 Drance SM. The early structural and functional disturbances of chronic open angle glaucoma. Ophthalmology 1985; 92 853-7.

6 Phelps CD, Corbett JJ. Migraine and low-tension glaucoma. A case-control study. Invest Ophthalmol Vis Sci 1985; 26: 1105-8.

7 Hotelling H. Analysis of a complex of statistical variables into principal components. F Educ Psychol 1933; 24: 417-41.

8 Morrison DF. Multivariate statistical methods. 2nd ed. New York: McGraw-Hill, 1976: 266-301.

9 Flury B, Riedwyl H. Multivariate statistics: a practical approach. London: Chapman and Hall, 1988: 181-233.

10 Flammer J, Drance SM, Augustiny L, Funkhauser A Quantification of glaucomatous visual field defects with automatic perimetry. Invest Ophthalmol Vis Sci 1985; 26: 176-81.

11 Drance SM, Douglas GR, Wijsman K, Schulzer M, Britton RJ. Response of blood flow to warm and cold in normal and low-tension glaucoma patients. Am f Ophthalmol 1988; 105: 35-9.

12 Hoyng PFJ, Greve EL, Frederikse K, Geijssen C, Oosting H. Platelet aggregation and glaucoma. Doc Ophthalmol 1985; 61: 167-73.

13 Meade TW, North WRS, Chakrabarti R, Stirling Y, Haines AP, Thompson SG. Haemosatic function and cardivascula death: early results of a prospective study. Lancet 1980; $i$ $1050-4$

14 Iatridis SG, Ferguson JH. Active Hageman factor: a plasma lysokinase of the human fibrinolytic system. $f$ Clin Invest 1962; 41: 1277-87. 